\title{
EFECTO DE UNA QUEMA CONTROLADA EN LOS ARTRÓPODOS EPÍGEOS DE PASTURAS EN LA SAIS TÚPAC AMARU, JUNÍN - PERÚ
}

\section{EFFECT OF CONTROLLED BURNING ON EPIGEAL ARTHROPODS IN TUPAC AMARU SAIS GRASSLANDS, JUNIN, PERU}

\author{
Liz Z. Castañeda Córdova ${ }^{1}$, Germán Arellano Cruz $^{2}$ y Edgar Sánchez Infantas ${ }^{1}$
}

\begin{abstract}
Resumen
El presente estudio describe el efecto de una quema controlada en la comunidad de artrópodos epígeos en los pastizales de la Sociedad Agrícola de Interés Social (SAIS) Túpac Amaru, Junín. La artropodofauna fue muestreada desde el final de la época seca (Agosto 2001) hasta el inicio de la siguiente época seca (Junio 2002), empleando trampas pitfall. La quema fue realizada a inicios del mes de Octubre (inicio de la época de lluvias), mediante una quema prescrita denominada quema frontal, a favor del viento. La parcela fue denominada Zona Quemada (ZQ) y fue comparada con otra parcela control denominada Zona No Quemada (ZNQ). El efecto de la quema en la comunidad de artrópodos fue evaluado analizando las variables comunitarias (abundancia, equidad, riqueza, diversidad y composición) a nivel de las morfoespecies separando los macroartrópodos y los microartrópodos y los grupos funcionales (abundancia y riqueza). La Clase Taxonómica más abundante fue Collembola con el $59 \%$, seguida por Insecta con el $28 \%$; el grupo funcional más abundante fue el detrítivoro. Las variables comunitarias fueron influenciadas por la dominancia marcada de la morfoespecie Entomobryidae-01 antes de la quema, lo cual generó una disminución en la diversidad. Luego de la quema se dieron diferencias significativas en la abundancia y la diversidad alfa promedio de la artropodofauna $(\mathrm{p}<0.05)$, con relación a los registros previos a la quema; sin embargo estas diferencias se dieron en ambas parcelas sugiriendo un efecto estacional. De todos los agrupamientos analizados, solo los grupos funcionales de los macroartrópodos parasitoides y los microartrópodos depredadores presentaron un efecto ante la quema.
\end{abstract}

Palabras clave: Artrópodos epígeos, quema controlada, SAIS Túpac Amaru, pasturas, trampas de caída, grupos funcionales

\begin{abstract}
The present study describes the effect of controlled burning (head fire) in epigeal arthropod assemblages in Junin grasslands. Arthropods were sampled from the end of the dry season (August 2001) to the beginning of the following dry season (June 2002), using pitfall traps. The burning experiment was carried in the first days of October (beginning of the rain season), using Head Fire Burnings (in favour of the wind). The plot was named Burnt Zone (ZQ) and was compared with a control plot named Non Burnt Zone (ZNQ). Burning effects in the arthropod assemblages were assessed analyzing community variables (abundance, equitability, richness, diversity and composition) at morphospecies level separating macroarthropods, microarthropods and functional groups (abundance and richness). The class that was most abundant was Collembola with $59 \%$, followed by Insect with $28 \%$; and the most abundant functional group comprised detritivores. Community variables were influenced by marked dominance of Entomobryidae-01 morphospecies before burning, which generated a decrease in diversity. Student's t-test was used and significant differences were found in the abundance and the diversity average alpha of the arthropods $(\mathrm{P}<0.05)$ before and after the burning, nevertheless these differences were found in both plots suggesting a seasonal effect. Of all the groupings analyzed only the functional groups of the macroarthropods parasitoides and the microarthropods predators presented an effect caused by the burning.
\end{abstract}

Key words: Epigeal arthropods, prescribed burning, Tupac Amaru SAIS, grasslands, pitfall traps, functional groups

\section{Introducción}

De acuerdo a la Estadística Agraria en el Perú, existen 18800000 ha de praderas nativas en la zona altoandina. Su importancia es innegable pues soportan el $86 \%$ de la ganadería nacional. Se estima que las áreas óptimas para el pastoreo son de 10576000 ha y el resto son áreas de protección (Flores, 1989). Estos pastizales deben ser manejados adecuadamente para 
asegurar la disponibilidad de alimento para el ganado. Un método de manejo empleado comúnmente por los pobladores andinos es la quema. La ONERN (1976), menciona que se realizan periódicamente quemas de estas pasturas con el objeto de permitir el aprovechamiento de la vegetación de piso por el ganado ovino y la obtención de un rebrote más suculento y tierno para el ganado vacuno (Florez et al., 1972).

Komarek (1962), sostiene que el fuego es una gran fuerza rejuvenecedora de la naturaleza sin la cual la sucesión vegetal y animal sería retardada de modo tal que la tierra estaría cubierta de comunidades decadentes. En realidad se trata del conocido hecho de que los ecosistemas, mientras más se acercan al clímax, al tiempo que incrementan su organización disminuyen su productividad neta, con lo que la cosecha que de ellos se puede extraer también disminuye sensiblemente. El efecto que esto tiene sobre las comunidades faunísticas presentes en las praderas nativas ha sido poco estudiado. Existe una escasez de información con respecto al efecto del fuego en la fauna y en especial a la artropodofauna que habita los ecosistemas de la puna.

Heyward \& Tissot (1936), citados por Wright \& Bailey (1982), señalan que el fuego puede disminuir la disponibilidad de alimento y la magnitud de este depende de la intensidad del fuego. Por lo menos disminuye de tres a diez veces sus abundancias y requiere de tres a cinco años para volver a los niveles anteriores. Un patrón similar se da en la respuesta de los pastizales del sudeste de Idaho, donde las abundancias relativas de hormigas y escarabajos requirieron de tres a cinco años para regresar a los niveles poblaciones existentes antes de la quema (Nelle et al., 2000). En el Bosque Nacional de Osceola (Florida) se realizaron quemas prescritas en invierno, donde las poblaciones requirieron más de 4 años para recuperarse por lo que se planteó incluir áreas de exclusión al fuego (Hanula \& Wade, 2003). Contradictoriamente en praderas altamente fragmentadas de Chicago, la rotación de la quema sugiere una contribución a la protección de varias especies de insectos en peligro (Panzer \& Schwartz, 2000) y en bosques de coníferas las quemas ribereñas estarían beneficiando la diversidad de mariposas (Huntzinger, 2003).

La quema (fuego de baja intensidad) empleada en los bosques secos de eucaliptos en el Sudeste de Australia también afecta de manera drástica a los invertebrados reduciendo significativamente el número de arácnidos, ácaros, pseudoescorpiones, colémbolas, hemípteros, coleópteros, hormigas y larvas de insectos que habitan en la hojarasca (York, 1999). Sin embargo las cucarachas (Blattodea), grillos y saltamontes (Orthoptera), inclusive los arácnidos fueron evaluados en un ecosistema similar en el Oeste de Australia, presentando una alta resiliencia a la tala y a la quema. Los cambios en la estructura de la comunidad causada por las perturbaciones impuestas fueron mínimas y por corto tiempo (Abbott et al., 2003).

Para evaluar estos cambios producidos por las perturbaciones en un ecosistema, se debe tomar en cuenta los diferentes niveles organizacionales que lo integran y los artrópodos han sido ampliamente reconocidos por su importancia dentro del ecosistema. Según Finnamore (1996), el 64\% de la diversidad global faunística esta constituida por los mismos, constituyendo la infraestructura invisible que dirige la dinámica del ecosistema, por lo tanto es una importante fuente de información, con una resolución muy fina al medir las perturbaciones, pudiendo ser complementada con la de otros organismos. Por lo expuesto anteriormente los artrópodos son estudiados debido a la disponibilidad de los mismos en áreas pequeñas, su relación con la vegetación (productividad primaria) y su respuesta rápida ante los cambios. Esta investigación tiene el objetivo de evaluar el efecto de la quema en la artropodofauna de los pastizales de la sierra central de nuestro país.

\section{Materiales y Métodos \\ Área de estudio}

El presente estudio se realizó en la zona denominada Champacancha (Lat. Sur $11^{\circ} 55^{\prime}$, Long. Oeste $75^{\circ} 45^{\prime}$ y tiene una altitud de $4100 \mathrm{msnm}$ ), ubicada en la unidad productiva ganadera Pachacayo, en el distrito de Canchayllo y la provincia de Jauja. Se delimitaron dos parcelas de $900 \mathrm{~m}^{2}$ cada una en las cuales se aplicaron dos tratamientos: la Zona Quemada (ZQ) y la Zona No Quemada (ZNQ), con el objetivo de comparar una zona en su estado natural con otra zona perturbada. Esta área pertenece a una muestra representativa de la asociación agrostológica Festuchetum-Calamagrosetum.

\section{Método de Quema}

La quema se llevó a cabo a inicios del mes de Octubre (inicio de época de lluvias), esto permitió una recuperación rápida de los pastizales debido a la precipitación. Además, quemar en esta época permite la protección de la semilla botánica mediante la ligera humedad y la reducción del arrastre de cenizas especialmente por el viento (Florez et al., 1972). En una parcela cuadrada se llevó a cabo una quema frontal a favor del viento (Head Fire), La quema duró aproximadamente 2 horas, los pastizales tuvieron una buena recuperación después de dos semanas y mostraron una amplia extensión de rebrotes luego de dos meses (Torres, 2004).

\section{Muestreos}

La mejor forma de saber como afecta una perturbación al ambiente, es evaluando el área estudiada antes de la perturbación, en este caso se 
realizaron ocho muestreos de campo, de las cuales dos muestreos fueron antes de la quema, los meses evaluados antes de la quema fueron Agosto-2001 (época seca) y Octubre-2001 (inicio de lluvias). La quema se realizó el 9 de Octubre del 2001, después de dos semanas de la quema se realizó el tercer muestreo (Octubre-2001), los siguientes dos muestreos se llevaron a cabo después de un mes (Noviembre-2001 y Diciembre-2001; época de lluvias), el sexto muestreo (Febrero-2002; época de lluvias), el séptimo muestreo (Abril-2002; inicio de época seca) y el octavo muestreo (Junio-2002; época seca). Las evaluaciones se realizaron en los meses representativos para la estacionalidad anual. Se realizó un muestreo sistemático; fueron seis puntos o estaciones para cada zona, en cada punto se colocaron tres trampas de caída.

Las trampas empleadas fueron envases de plástico transparentes de $6 \mathrm{~cm}$ de diámetro, $8.5 \mathrm{~cm}$ de profundidad y una capacidad de $250 \mathrm{~cm}^{3}$, enterrados al ras del suelo y llenados casi completamente con una mezcla de agua con etilenglicol al $2 \%$, a la cual se añadió 1 gr de detergente para reducir la tensión superficial. El tamaño de la unidad muestral (TUM) fue de tres trampas por estación con un esfuerzo temporal de tres días - aproximadamente 72 horas -, esfuerzo determinado en el primer muestreo, a partir de la elaboración de espectros de diversidad (Castañeda, 2007).

Los artrópodos de cada muestra fueron extraídos y clasificados bajo la denominación de morfoespecies (Oliver \& Beattie, 1996; Derraik et al., 2002). Los artrópodos fueron determinados al nivel de familia y en algunos casos a subfamilia, utilizando diferentes claves taxonómicas. Además se separaron los Macroartrópodos de los Microartrópodos. Esta separación de la artropodofauna se debe a que el grupo denominado Microartrópodos presenta características similares tanto en el tamaño que poseen como en el hábitat que ocupan, siendo artrópodos pequeños que habitan el suelo e interaccionan en este sustrato formando una comunidad (Castañeda, 2007). La clasificación trófica de acuerdo al recurso alimentario, también nos permitió organizar a la artropodofauna en grupos funcionales (Davic, 2003). Una vez concluida las labores de determinación los especimenes fueron depositados en el Laboratorio de Ecología de Procesos - Departamento de Biología de la Universidad Nacional Agraria La Molina (UNALM).

\section{Análisis de los datos}

La evaluación del efecto de la quema en la comunidad de artrópodos se realizó mediante la medición de variables comunitarias agrupando a las morfoespecies, a los macroartrópodos y a los microartrópodos; y a los grupos funcionales. Las variables comunitarias estudiadas fueron la abundancia, la equidad, la riqueza y la diversidad. Los conceptos de estas variables se detallan a continuación: la abundancia es el número de individuos capturados, la riqueza es el número de morfoespecies, la diversidad se calculó a partir de las abundancias de las morfoespecies empleando el índice de diversidad de Shannon (Magurran, 1988). La diversidad fue calculada para cada estación (diversidad alfa) y en cada parcela se calculó la diversidad $\alpha$ promedio para cada muestreo, con sus respectivos intervalos de confianza obtenidos a partir de la distribución t de Student (Duncan et al., 1978). También se estimó la diversidad gamma $(\gamma)$ y los intervalos de confianza se obtuvieron mediante el programa PAST (Hammer et al., 2001), mediante una técnica de bootstrap a un nivel de $95 \%$ de confianza. Para determinar diferencias estadísticas significativas $(\alpha=0.05)$ entre el tratamiento ZQ y ZNQ, se empleó la prueba t de Student para muestras pareadas entre los resultados de abundancia y diversidad $\alpha$ promedio de la artropodofauna antes y después de la quema.

Los cambios espaciales y temporales de los artrópodos se evaluaron a través de las siguientes medidas: la distribución de abundancias y el análisis de cluster. Para la distribución de abundancias se emplean histogramas, los cuales permiten visualizar los distintos tipos de distribución y el conocimiento de las especies dominantes para cada muestreo; los histogramas se realizaron a nivel de los macroartrópodos, los microartrópodos e incluyendo a todas las morfoespecies. Se tipifica las distribuciones de abundancia siguiendo el enfoque de Giraldo (2002), siendo el siguiente:

(1) Dominancia Marcada, cuando una de las especies (especie dominante) se destaca claramente de las demás por su mayor abundancia.

(2) Dominancia Exagerada, caso particular de dominancia marcada en el cual la especie dominante comprende más del $80 \%$ de la abundancia total.

(3) Dominancia Compartida, cuando dos especies se destacan de las demás por sus mayores abundancias

(4) Distribución Equitativa cuando no hay especies que se destaquen notoriamente por sus mayores abundancias.

El análisis de cluster se empleó para estaciones y para muestreos. Este tipo de análisis permite comparar simultáneamente una serie de muestras e ilustrar las relaciones existentes entre ellas, usando el Índice de similitud de Raup-Crick tomando en cuenta la ausencia y la presencia de las especies, y el índice de similitud de Morisita que toma en cuenta la abundancia (Krebs, 1989). Ambos tipos de análisis se realizaron empleando el programa PAST (Hammer et al., 2001). 
Los artrópodos fueron agrupados en 7 grupos funcionales mediante la elaboración de una clasificación trófica: los detrítivoros, los fitófagos, los micetófagos, los parasitoides, los depredadores, los saprófagos y los ectoparásitos. Dichas categorías se obtuvieron a partir de las preferencias predominantes dentro de cada familia. La dieta de los coleópteros fue obtenida de Crowson (1981) y el carábido Notiobia peruviana fue definido fitófago por su predilección por las semillas en las pruebas realizadas en laboratorio (Loza \& Apaza, 2001). La categorización anterior permitió organizar a la artropodofauna en grupos funcionales, permitiendo evaluar en algunos grupos una variación luego de la quema.

Se analizó la similitud de las comunidades de la artropodofauna entre ambas zonas, a diferentes niveles. La comparación entre toda la artropodofauna (primer nivel de agrupación), se realizó empleando la mediana de los valores que proporciona el Índice de Similitud de Raup-Crick y el Índice de Similitud de Morisita. La comparación entre Macroartrópodos y Microartrópodos (segundo nivel de agrupación), se realizó con el coeficiente de correlación $r$ de Spearman. La comparación entre los grupos funcionales (tercer nivel de agrupación) con y sin Entomobryidae-01 se realizó con el coeficiente de correlación $\mathrm{r}$ de Pearson. Todos los índices de correlación fueron calculados con el programa PAST (Hammer et al., 2001).

\section{Resultados y discusión}

Se registraron un total de 17671 individuos, de los cuales 8723 individuos corresponden a la ZNQ y 8 948 individuos corresponden a la ZQ, distribuidos en 16 ordenes, 85 familias determinadas, 2 familias indeterminadas y 239 morfo(especies). De las Clases presentes la más abundante fue Collembola con el 59 $\%$, seguida por Insecta $28 \%$ y por último Arachnida con el 13\%. Los Ordenes más abundantes fueron Arthropleona, Hemiptera, Symphypleona, con el 46.3 $\%, 13.0 \%, 12.7 \%$ del total de individuos capturados respectivamente. Las familias más abundantes han sido: Entomobryidae con $44.15 \%$, Sminthuridae con $12.7 \%$ y Cicadellidae con $10.29 \%$. Las abundancias de los Ordenes Taxonómicos de ambas zonas se presentan en el Anexo 1.

Al evaluar la abundancia y su variación, se observaron los mayores valores para la $\mathrm{ZQ}$ a inicios de octubre antes de la quema, y en la ZNQ en el mes de febrero (Figura 1), al parecer estos patrones están relacionadas con la precipitación. Este parámetro tiene una relación inversa con la $Z Q$ y una relación directa con la ZNQ. En el estudio de Dennis et al. (1997) se observa una relación directa de la abundancia de artrópodos con la estructura vertical de la vegetación, este patrón se estaría expresando en la ZNQ. En el mes de agosto la abundancia entre las dos parcelas difiere, sin embargo hay que considerar que la morfoespecie Entomobryidae-01 esta generando un efecto perturbador (Castañeda, 2007). Al analizar la abundancia sin esta morfoespecie (Figura 1) al inicio y al final de los muestreos ambas zonas son similares, sugiriendo que la diferencia observada en el segundo y sexto muestreo podría deberse a un efecto estacional.

La equidad muestra una relación lineal directa con la diversidad gamma; esta estrecha relación, es consecuencia de la distribución de abundancias denominada Dominancia Marcada, la cual parece ser el patrón típico de la comunidad de la artropododofauna. A partir del muestreo de Noviembre hasta el último muestreo esta variable no se diferencia, mostrando que ambas comunidades se vuelven equitativamente similares. Algo parecido se da con la riqueza; luego de la quema, la $\mathrm{ZQ}$ incrementa el número de especies debido a la aparición de especies oportunistas. Las dos parcelas están en un proceso de sucesión natural, esto se refleja en la aparición de nuevas especies en cada muestreo y en la similitud entre ambas en el último muestreo (Figura 2). La variación temporal de la diversidad alfa promedio también se evalúa con y sin Entomobryidae01, observándose en los muestreos antes de la quema que cuando esta presente Entomobryidae-01 las diversidades son diferentes para ambas zonas; luego de la quema las diversidades entre las zonas se vuelven similares. La variación temporal de la diversidad alfa promedio sin Entomobryidae-01 no muestra diferencias entre las zonas (Figura 3).

En la variación temporal de la diversidad gamma (Figura 4) para ambas zonas se presentó un descenso marcado a inicios de Octubre, que estuvo relacionado a la ocurrencia de Dominancias Marcadas con valores muy cercanos al $80 \%$ de Entomobryidae-01 y a un incremento a fines de Octubre debido a una Dominancia Marcada (con valores entre 30 y $40 \%$ de Entomobryidae-01) asociada a una mayor equidad de las otras especies. Si se analiza la diversidad gamma temporal luego de la quema se observa un incremento de la diversidad para ambas zonas.

Al parecer la abundancia y la diversidad $\alpha$ promedio a través del tiempo no mostraron un efecto debido a la quema, lo cual refuerza la sugerencia de estar observando un efecto estacional, debido a que cuando se dieron cambios drásticos se dieron en ambas zonas, es decir se encontraron diferencias significativas empleando la prueba $\mathrm{t}$ de Student para muestras pareadas en ambas zonas (Tabla $1 ; \alpha=0.05$ ). Las diferencias temporales dentro de ZQ y ZNQ son mayores que las diferencias espaciales entre estas dos parcelas.

$\mathrm{Si}$ bien la diversidad (excepto la diversidad gamma) no mostró comportamientos diferentes entre ambas zonas, esta variable permite visualizar procesos y patrones que se dan en la comunidad de la artropodofauna. Es así como la variación temporal de la abundancia, riqueza, equidad y las diversidades alfa 
promedio y gamma de la artropodofauna dan cuenta de un proceso de sucesión inducido por la estacionalidad durante el año de evaluación, solo que cada variable en la ZQ requiere tiempos diferentes para volver a valores similares a la ZNQ. Sin embargo al analizar la diversidad gamma temporal luego de la quema se observa un cambio en el patrón debido a la sobreposición de los valores de diversidad de la ZQ frente a la ZNQ, lo cual sugiere que la quema esta incrementando ligeramente la diversidad, es decir, el efecto inicial de la quema fue elevar la diversidad con respecto a su nivel normal, por lo cual podemos considerarlo como una perturbación (Huston, 1994). Según Margalef (1991), si las perturbaciones no son frecuentes, pueden generar un incremento de la diversidad, debido a que a los efectos iniciales sigue un ajuste posterior que conduce a un aumento gradual de masa y la disminución de la tasa de renovación. Esta respuesta es reafirmada por Huston (1994) que manifiesta que las diversidades más altas ocurren a frecuencias intermedias de perturbación, mientras que las diversidades más bajas lo hacen a frecuencias muy bajas o muy altas de perturbación; se estaría asociando este último comportamiento al efecto estacional, el cual genera al aparecer una alta perturbación en la comunidad de los artrópodos.

Las distribuciones de abundancias asociadas a cada muestreo para las morfoespecies, Macroartrópodos y Microartrópodos se muestran en la Tabla 2. Se observó que la Dominancia Marcada ha sido el patrón predominante siendo las únicas excepciones para la ZQ el muestreo de Abril-2002 (Dominancia Compartida) y para la ZNQ en los meses de Febrero-2002 (Dominancia Compartida) y Abril2002 (Dominancia Compartida), lo cual nos lleva a pensar que las Dominancias Marcadas son la condición típica de la comunidad y que la alteración provocada por la quema no condujo a cambios en la distribución de las abundancias. En los Macroartrópodos la Dominancia exagerada no se dio para ningún muestreo, sin embargo es interesante observar que la ZNQ tiene como patrón predominante la Dominancia Marcada y la ZQ tiene como patrón predominante la Distribución Equitativa, lo cual nos lleva a pensar que las Dominancias Marcadas son la condición típica de la comunidad y las alteraciones provocadas por la estacionalidad y la quema conducen a cambios en la distribución de sus abundancias.

Los Microartrópodos son numéricamente los más importantes de la fauna del suelo (Margalef, 1977). Es interesante apreciar que ambas zonas también tienen como patrón predominante la Dominancia Marcada, esto sugiere que es la condición típica de la comunidad, sin embargo es interesante apreciar que ambas zonas antes de la quema poseen una Dominancia Exagerada y luego de varios meses ambas zonas muestran una Dominancia Compartida, sugiriendo que la quema no afecta las distribuciones inmediatamente sino la estacionalidad, posiblemente la quema retrasa la Distribución Compartida en la ZQ (Abril-2002), dándose antes en la ZNQ (Febrero2002).

Empleando el análisis de clusters se evaluó la similitud de la composición de morfoespecies entre ambas zonas antes y después de la quema, considerando su ausencia y presencia (Figura 5). Antes de la quema a una distancia de enlace menor a 0.5 se distinguieron dos asociaciones, una asociación agrupando a las estaciones 5 y 6 de la ZNQ y otra agrupación con el resto de las estaciones, sin embargo luego de la quema al mismo nivel de asociación (0.5) las dos parcelas mostraron una gran similitud en su composición de especies, es decir, que la quema inicialmente estaría homogenizando la zona. Además se evaluaron todos los muestreos en ambas zonas, a una distancia de enlace menor a 0.5 no se observó diferencias en la abundancia y la presencia-ausencia de los artrópodos. Sin embargo se observaron agrupamientos de los muestreos asociados a la presencia de las especies por la estacionalidad, a distancias de enlaces mayores a 0.5 (Figura 6).

Analizando los grupos funcionales los detrítivoros superaron en abundancia a los demás grupos, debido a la gran abundancia de Entomobryidae-01. Los fitófagos presentaron el mayor número de especies y fueron segundos en abundancia. Con respecto al efecto de la quema en los grupos funcionales, se observaron efectos negativos así como efectos positivos en los macroartrópodos parasitoides y los microartrópodos depredadores respectivamente. Al parecer tanto la precipitación como la diversidad de estratos están muy relacionadas con la abundancia de estos dos grupos funcionales, los macrortrópodos parasitoides tanto en su abundancia absoluta y relativa, fluctúan luego de un mes de la quema, siendo más notorio al aumentar la precipitación. En la estación seca ambas parcelas se vuelven similares, probablemente la precipitación es un efecto estacional que al sumarse al efecto quema, permite al grupo de los parasitoides ser un indicador de estas perturbaciones (Figura 7). Los porcentajes de abundancia de los depredadores en la ZQ se incrementaron luego de la quema, hasta alcanzar el mayor ascenso en el mes de Noviembre, luego desciende gradualmente en los meses de Diciembre y Febrero, para después terminar siendo menor en los dos últimos muestreos, correspondientes a la época seca, al parecer la disminución de la precipitación genera un ligero efecto negativo en la $\mathrm{ZQ}$, que permite que regrese al comportamiento que tenía antes de la quema (Figura 8)

Además al evaluar la similitud de la comunidad de la artropodofauna, a nivel de su abundancia y su composición luego de la perturbación generada por la quema, se encontró que la quema no afectó la correlación entre ambas zonas, sin embargo algunos 
descensos en la correlación reforzarían el efecto estacional (Figura 9). En la similitud temporal de los Macroartrópodos y los Microartrópodos (Figura 10), luego de la quema se puede observar que la similitud oscila entre un coeficiente de correlación $r$ de Spearman de 0.6 y 0.9 , sugiriendo que la quema no afectó la similitud entre la comunidad de Microartrópodos (Tabla 3). Con respecto a los Macroartrópodos los valores de $\mathrm{r}$ en todas las muestreos han sido bajos, es decir que ambas parcelas no son muy parecidas entre sí para este grupo. Además antes y después de la quema no se dan cambios importantes en $r$, los datos significativos para estas dos muestreos se muestran en la Tabla 3, sugiriendo que la quema no afecta la similitud entre la comunidad de Macroartrópodos. Esto corrobora lo sugerido cuando se analizan todas las morfoespecies. Al comparar las comunidades en función a sus grupos funcionales con el coeficiente de correlación $r$ de Pearson no se dan variaciones importantes ni antes ni después de la quema (Figura 11); todos los valores de $r$ son significativos (Tabla 4). Se realizó el mismo análisis sin tomar en cuenta a Entomobryidae-01 (Figura 11), tampoco las variaciones fueron drásticas (Tabla 4). Estos tres niveles de análisis muestran que no habría un efecto drástico de la quema en las comunidades de artrópodos.

El efecto leve de la quema observado en la presente investigación puede deberse a alguna de los siguientes planteamientos o a la interacción de las mismos. El primer planteamiento es debido a la época en la cual se realizó la quema, sugiriendo que una quema controlada a favor del viento realizada a inicios de la época de lluvias, no genera un impacto fuerte. El segundo planteamiento está relacionado al tamaño de las parcelas, probablemente $900 \mathrm{~m}^{2}$ es un área lo suficientemente pequeña para ser colonizada rápidamente y no tener los efectos negativos que se estarían produciendo en una quema de grandes extensiones. El tercer planteamiento sugiere que la artropodofauna del ecosistema de la puna ha coexistido con el fuego, durante mucho tiempo. Por lo tanto como Huston (1994) indica los ecosistemas con frecuentes quemas tienden a exhibir al menos conspicuas respuestas ecológicas. En tales ecosistemas los patrones predominantes de la composición de especies y diversidad del paisaje no resulta de la variación en la frecuencia o en la intensidad del fuego, sino de la variación de las condiciones ambientales que influencian la productividad de la planta y de su tasa de desplazamiento y recuperación. En la presente investigación se ha podido observar este patrón en las diferentes variables evaluadas, es decir, son las condiciones ambientales las que tienen un mayor efecto en la productividad de los pastizales y en la comunidad de artrópodos. El cuarto planteamiento es que se debió también evaluar a los artrópodos del estrato herbáceo, porque son los primeros afectados por la quema.

La respuesta de los ecosistemas frente a las perturbaciones está asociada al concepto de resiliencia. La sostenibilidad del sistema involucra el mantenimiento de la funcionalidad del mismo luego de una perturbación o el mantenimiento de los elementos necesarios para renovarlo o reorganizarlo, esta habilidad es denominada resiliencia (Walker et al., 2002). Asimismo, en el contexto del manejo adaptativo, la resiliencia se define como la habilidad de un sistema para absorber cambio y variación sin saltar a un estado diferente donde las variables y procesos que controlan su estructura y comportamiento cambien súbitamente (Holling, 1996). Considerando el concepto de manejo adaptativo se ha construido un modelo conceptual, tratando de interpretar el desarrollo de los procesos ecológicos de la unidad productiva de Pachacayo. Existe un ciclo adaptativo en el modelo correspondiendo a la escala local, el cual se puede explicar debido a que se desarrolla sobre un ciclo anual. Los otros ciclos (al nivel de microcuencas y regiones) requieren una mayor información para conocer el comportamiento de sus variables en las diferentes fases. Todos estos ciclos presentan una cierta regularidad, la misma que para el nivel más pequeño - motivo del presente estudio - se puede describir en los siguientes términos: existe un proceso de crecimiento (r) y otro de acumulación estacional de biomasa vegetal $(\mathrm{K})$. Parte de esta biomasa es retirada como consecuencia de la actividad ganadera, pero hay un excedente de biomasa seca. Debido a ello se emplea la quema del pastizal como mecanismo para retirarla, lo que da paso a una liberación de nutrientes (fase $\Omega$ ) y posiblemente se da una pérdida de estos nutrientes (fase $\boldsymbol{\alpha}$ ), pero leve no drástica, por lo tanto esto permitiría sugerir que en el caso de los artrópodos no se genera una alta perdida de diversidad. De este modo, esto estaría reforzando la tercera alternativa de explicación - o tercer planteamiento - esbozado líneas arriba y que sugiere que posiblemente estos artrópodos tienen mucho tiempo co-existiendo con el fuego y han desarrollado respuestas adaptativas, es decir, que este ciclo se cierra tan rápido que no afecta al sistema. Sin embargo hay que considerar la frecuencia y la época en la que se realiza la quema, además la incorporación de la variable climática, la cual sumada a la quema cuando se dan condiciones climáticas adversas si pueden generar una gran disminución de la diversidad de los artrópodos.

\section{Conclusiones}

Las conclusiones de este trabajo permiten obtener un alcance sobre el efecto de las quemas en la artropodofauna, el cual no se lograría con solo una caracterización de áreas quemadas. En síntesis los resultados obtenidos indican que la quema controlada 
de pastizales no tiene un efecto negativo en la comunidad de artrópodos. La quema, es un tipo de perturbación que está incrementando ligeramente la diversidad. Posiblemente esta comunidad tiene mucho tiempo co-evolucionando con el fuego y han desarrollado respuestas adaptativas para que el sistema no se afecte. Las comunidades de artrópodos de la puna estarían entonces presentando dos tipos de perturbaciones: (1) la quema, la cual sería una perturbación media que facilita diversidades más altas y (2) la estacionalidad, la cual sería una perturbación muy alta que manifiesta diversidades muy bajas, por lo que al aparecer es la estacionalidad la que genera una alta perturbación en la comunidad de los artrópodos.

\section{Agradecimientos}

Los autores agradecen al Centro Internacional de la Papa (CIP) por el apoyo financiero para los muestreos de campo. Al personal de la SAIS Túpac Amaru por las facilidades brindadas en todos los muestreos realizados. A Roberto Quiroz, Diana Torres, Raúl Castañeda, Mónica Maldonado, Zulema Quinteros, Alfredo Giraldo, Rianne Van Der Bom y todos los estudiantes de Biología que apoyaron en los muestreos de campo y en la fase de laboratorio.

\section{Literatura citada}

Abbott I., Burbidge T., Strehlow K., Mellican A. \& Wills A. 2003. Logging and burning impacts on cockroaches, crickets and grasshoppers, and spiders in Jarrah forest, Western Australia. Forest Ecology and Management. 174: 383-399.

Castañeda L. 2007. Caracterización y evaluación de la diversidad de la artropodofauna en pastizales bajo el efecto de una quema controlada en la SAIS Túpac Amaru. Tesis para optar el título de Bióloga-UNALM.

Crowson R.A. 1981. The Biology of the Coleoptera. London, Academic Press Inc.

Davic R.D. 2003. Linking Keystone Species and Functional Groups: A New Operational Definition of the Keystone Species Concept. Conservation Ecology. 7(1): r11.

Dennis P., Young M.R., Howard C.L. \& Gordon I.J. 1997. The response of epigeal beetles (Col:Carabidae, Staphylinidae) to varied grazing regimes on spatial Nardus stricta grasslands. Journal of Appplied Ecology. 34: 433-443.

Derraik J.G., Cross G.P., Dickinson K.J.M., Sirvid P., Barrat B.I.P. \& Patrick B.H. 2002. Arthropod morphospecies versus taxonomic species: a case study with Araneae, Coleoptera and Lepidoptera. Conservation Biology. 16(4): $1015-1023$.

Duncan R., Knapp R. \& Clinton M. 1978. Bioestadística. México D.F., Nueva Editorial Interamericana S.A.

Finnamore A.T. 1996. The advantages of using arthropods for ecosystem management. Abrief prepared on behalf of the Biological Survey of Canada. : 1-11.

Flores A. 1989. Los Pastizales en el Perú. Revista del INIA. 1(3): 15.
Florez A., Segura M \& Gross H. 1972. Efecto de la quema sobre pastizales naturales altos andinos. Programa de Forrajes. UNALM. Boletín técnico. 13: 1-18.

Giraldo A. 2002. Análisis de los patrones de variación espacio-temporal de las poblaciones de coleópteros en la Reserva Nacional de Lachay durante el periodo 19982001. Tesis para optar el título de Biólogo- UNALM.

Hammer Ø., Harper D.A.T. \& Ryan P. D. 2001. PAST: Paleontological Statistics Software Package for Education and Data Analysis [en línea] Palaeontología Electrónica. 2001. 4(1). 9pp. http: //palaeoelectronica.org/2001_1/ past/ issue1_01.htm>

Hanula J \& Wade D. 2003. Influence of long-term dormantseason burning and fire exclusión on ground-dwelling arthropod populations in longleaf pine flatswoods ecosystems. Forest Ecology and Management. 175: 163184.

Heyward F. \& Tissot A. 1936. Some changes in the soil fauna associated withforest fires in the longleaf pine

region.Ecology. 17: 659-666.

Holling C.S. 1996. Surprise for science, resilience for ecosystems, and incentives for people. Ecological Applications. 6(3): 733-735.

Huntzinger M. 2003.Effects of fire management practices on butterfly diversity in the forested western United States. Biological Conservation. 113: 1-12.

Huston M. 1994.Biological diversity the coexistence of species in changing landscapes. Cambridge University Press.

Krebs C.J. 1989. Ecological Methodology. University of British Columbia. Harper Collins Publishers.

Komarek E.V. 1962. Fire ecology proc. Lst. Ann. Tall Timbres Fire ecological conference. Tallahassee. Fla. : 95-107.

Loza A.L. \& Apaza A. 2001. Amplitud depredadora y preferencia de presa en tres especies de carábidos (Coleoptera) del altiplano de Puno, Perú. Revista Peruana de Entomología. 42: 73-78.

Magurran A. 1988. Diversidad Ecológica y su Medición. Bangor, Ediciones Vedra S.A. 1998.

Margalef R. 1977. Ecología. Barcelona-España, Ediciones Omega S.A.

Margalef R. 1991. Reflexiones sobre la diversidad y significado de su expresión cuantitativa. Diversidad biológica. : 105-114.

Nelle P.J., Reese K.P \& Connelly J.W. 2000. Long-term effects of fire on sage grouse habitat. Journal of Range Management. 53: 586-591.

Oliver I. \& Beattie A. 1996. Invertebrate morphospecies as surrogates for species: a case study. Conservation Biology. 10(1): 99-109.

ONERN (Oficina Nacional de Evaluación de Recursos Naturales). 1976. Inventario y Evaluación de los Recursos Naturales de la SAIS "Túpac Amaru”. ONERN. Lima-Perú.

Panzer R. \& Schwartz M. 2000.Effects of management on prairie insect species richness within a system of small highly fragmented reserves. Biological Conservation. 96: 363-369.

Torres D. 2004. Evaluación de la variación de las poblaciones microbianas bajo el efecto de una quema controlada en Champacancha-Pachacayo-SAIS Túpac Amaru. Tesis para optar el título de Bióloga-UNALM. 
Walker B., Carpenter S., Anderies J., Abel N., Cumming G., Janssen M., Lebel L., Norberg J., Peterson G.D. \& Pritchard R. 2002. Resilience management in socialecological systems: a working hypothesis for a participatory approach. Conservation Ecology. 6(1): 14.

Wright H.A. \& Bailey A.W. 1982. Fire Ecology. United

States and Southern Canada. John Wiley \& Sons. New

York.
York A. 1999. Long-term effects of frequent low-intensity burning on the abundance of litter-dwelling invertebrates in coastal blackbutt forests of southeastern Australia. Journal of Insect Conservation. 3: 191-199.

Tablas y figuras y anexo citados en el texto.

Tabla 1: Prueba t aplicada a la diversidad $\alpha$ y la abundancia de la artropodofauna antes y después de la quema. Pachacayo.

(a) diversidad

\begin{tabular}{|c|c|c|c|c|c|c|c|c|}
\hline & \multicolumn{2}{|c|}{ Zona Quemada } & \multicolumn{2}{|c|}{ Zona No Quemada } & \multicolumn{2}{|c|}{ Zona Quemada } & \multicolumn{2}{|c|}{ Zona No Quemada } \\
\hline & Antes & Después & Antes & Después & Antes & Después & Antes & Después \\
\hline Media & 1.50 & 3.71 & 2.40 & 3.29 & 477.5 & 71.17 & 184.67 & 108.67 \\
\hline Varianza & 0.05 & 0.03 & 0.06 & 0.12 & 10900.7 & 486.57 & 1111.87 & 646.27 \\
\hline Observaciones & 6 & 6 & 6 & 6 & 6 & 6 & 6 & 6 \\
\hline Diferencia hipotética de las medias & 0 & & 0 & & 0 & & 0 & \\
\hline Grados de libertad & 5 & & 5 & & 5 & & 5 & \\
\hline Estadístico t & -20.85 & & -6.39 & & 11.58 & & 6.22 & \\
\hline $\mathrm{P}(\mathrm{T}<=\mathrm{t})$ dos colas & 0.000005 & & 0.001388 & & 0.00008 & & 0.0016 & \\
\hline Valor crítico de t (dos colas) & 2.57 & & 2.57 & & 2.57 & & 2.57 & \\
\hline
\end{tabular}

Tabla 2: Tipos de Distribución de las abundancias de las morfoespecies, macroartrópodos y microartrópodos en cada muestreo.

\begin{tabular}{|c|c|c|c|c|c|c|}
\hline Muestreos & Z. Q & Z.NQ & Z. Q & Z.NQ & Z. Q & Z.NQ \\
\hline Agosto 2001 (a.q) & DM & DM & DM & DC & DEX & DM \\
\hline Octubre 2001 (a.q) & DM & DM & DEQ & DEQ & DEX & DEX \\
\hline Octubre 2001 (d.q) & DM & DM & DEQ & DM & DM & DM \\
\hline Noviembre 2001 (d.q) & DM & DM & DEQ & DM & DM & DM \\
\hline Diciembre 2001 (d.q) & DM & DM & DC & DM & DM & DM \\
\hline Febrero 2002 (d.q) & DM & DC & DM & DC & DM & DC \\
\hline Abril 2002 (d.q) & DC & DC & DEQ & DC & DC & DC \\
\hline Junio 2002 (d.q) & DM & DM & DM & DM & DM & DM \\
\hline
\end{tabular}

$\mathrm{DM}=$ Dominancia Marcada $\quad \mathrm{DEQ}=$ Distribución Equitativa a.q = antes de quema

$\mathrm{DC}=$ Dominancia Compartida $\mathrm{DEX}=$ Dominancia exagerada d.q= después de quema

Tabla 3: Matriz de valores de probabilidad obtenidos mediante el Coeficiente de Correlación $r$ de Spearman para la comparación de Microartrópodos y Macroartrópodos. Los valores en negrita indican el grado de significación a un nivel de significación de $\mathrm{p}=0,05$.

(a) Microartrópodos

(b) Macroartrópodos

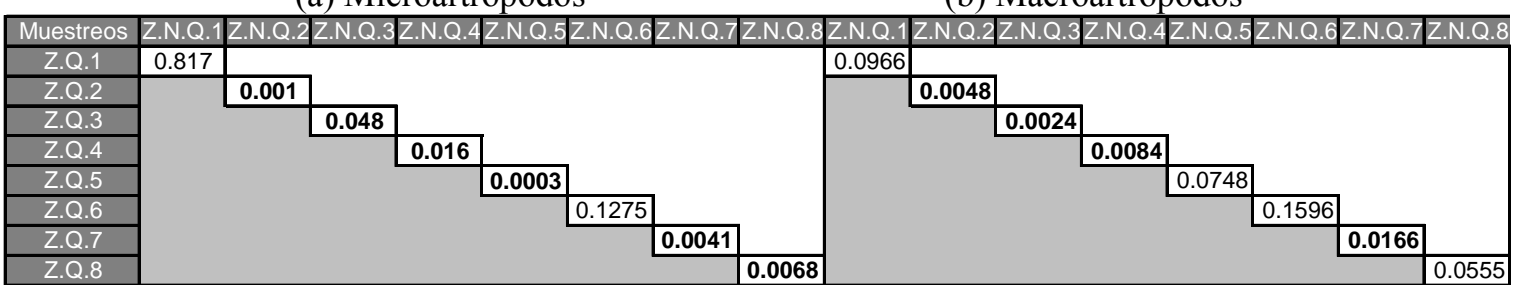


Figura 1. Evolución temporal de la abundancia de la Artropodofauna con y sin Entomobryidae-01. Agosto 2001 a Junio 2002. Pachacayo. IC ( $\mathrm{t}, \alpha=0.05)$.

(a) Con Entomobryidae-01

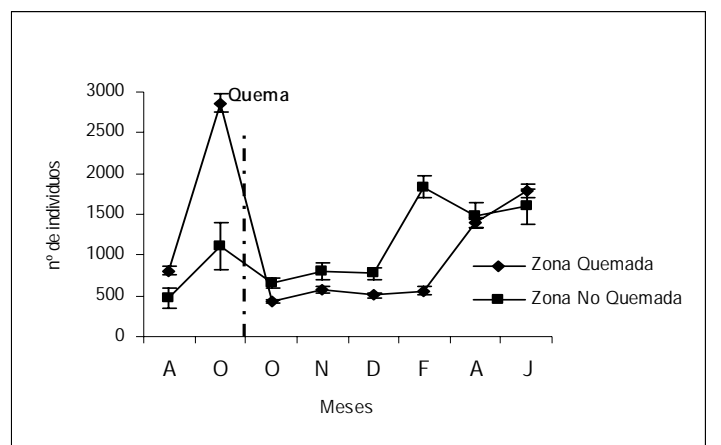

(b) Sin Entomobryidae-01

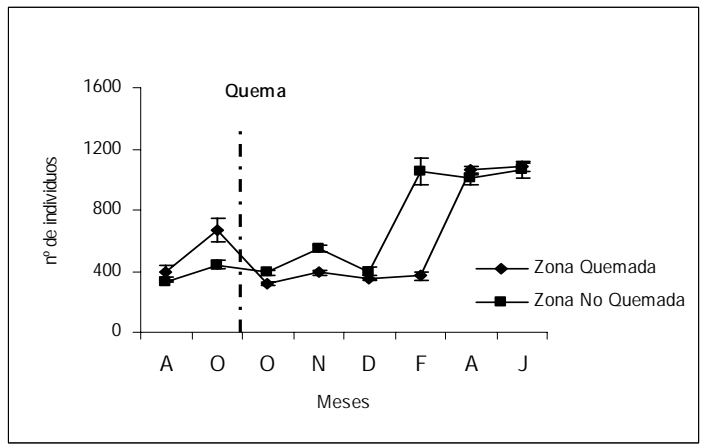

Figura 2. Evolución temporal de la equidad y la riqueza de la Artropodofauna. Agosto 2001 a Junio 2002. Pachacayo. IC (t, $\alpha=0.05)$.

(a) Equidad

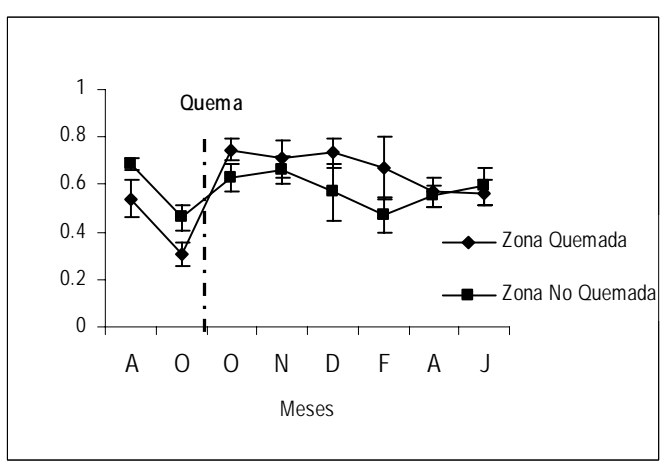

(b) Riqueza

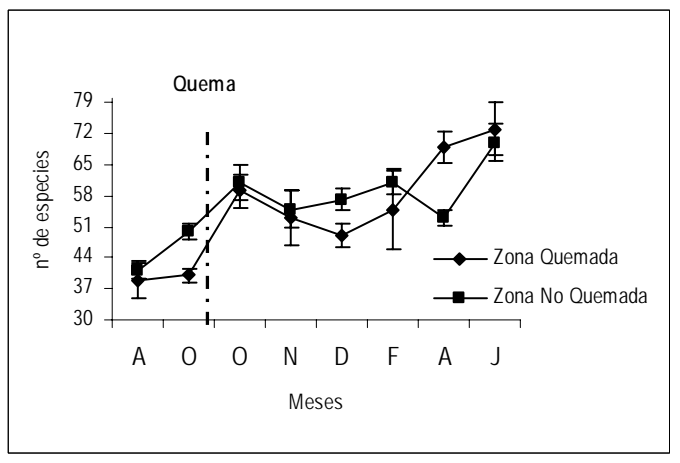

Figura 3. Evolución temporal de la diversidad alfa promedio de la Artropodofauna con y sin Eentomobryidae01. Agosto 2001 a Junio 2002. Pachacayo. IC (t, $\alpha=0.05)$.

(a) Con Entomobryidae-01

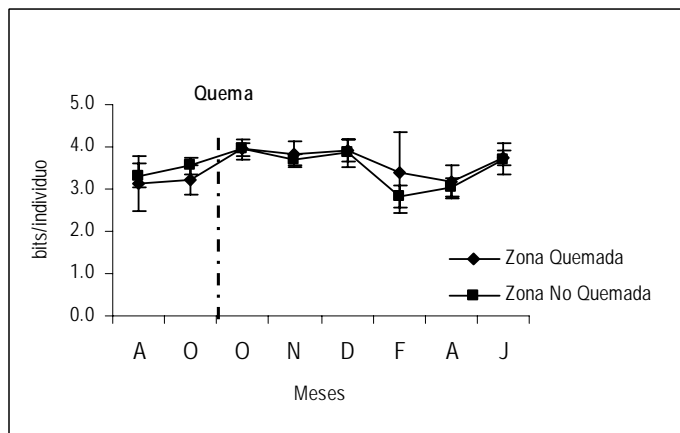

(b) Sin Entomobryidae-01

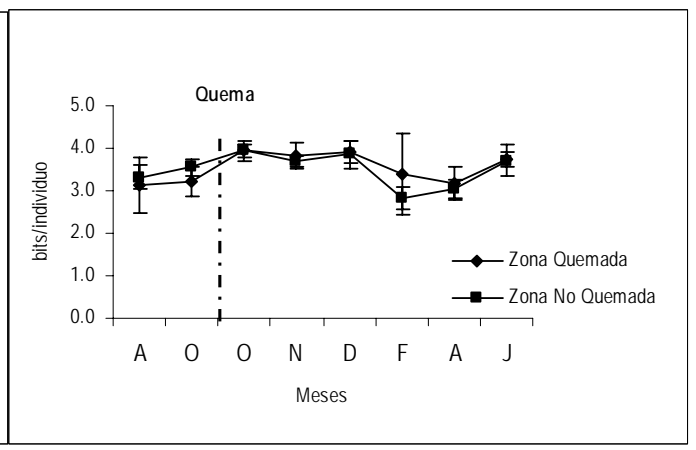


Figura 4. Evolución temporal de la diversidad gamma de la Artropodofauna. Agosto 2001 a Junio 2002. Pachacayo. IC ( $\mathrm{t}, \alpha=0.05)$.

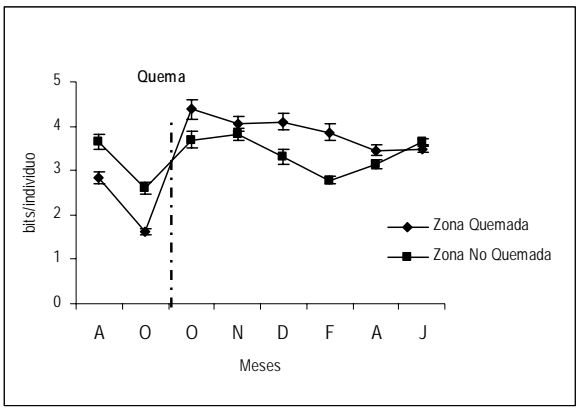

Figura 5. Dendrograma de datos de presencia-ausencia de artrópodos en Octubre 2001. Pachacayo. Índice de Similitud de Raup-Crick

(a) Antes de Quema

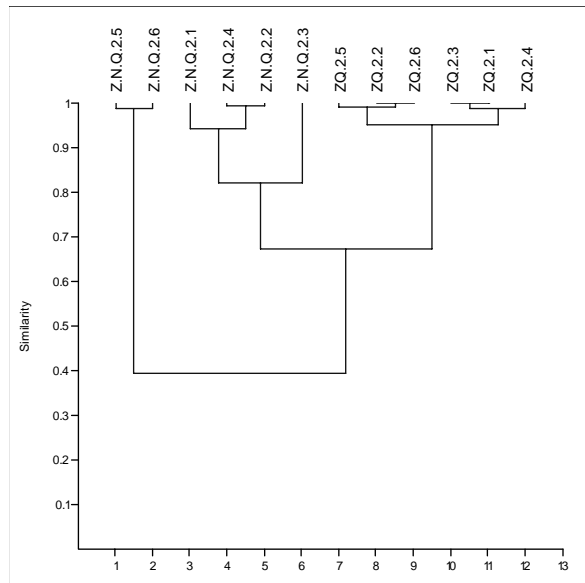

(b) Después de Quema

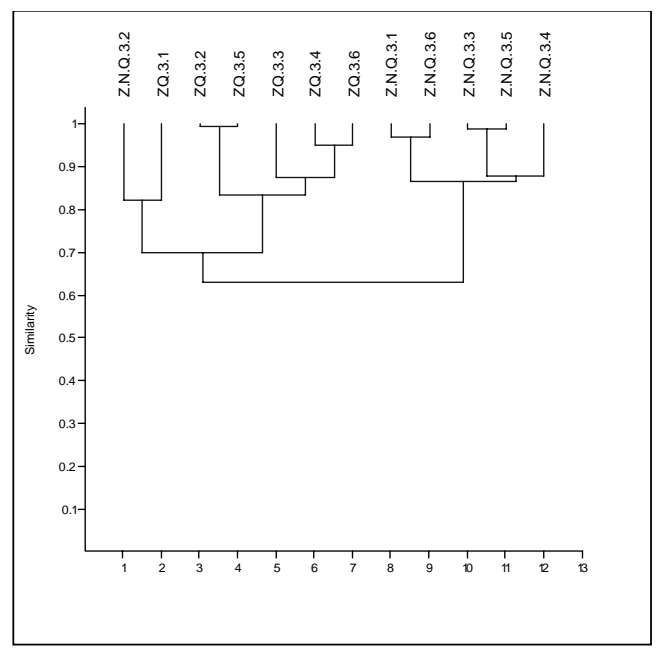

Figura 6. Dendrograma de los artrópodos en todos los muestreos. Pachacayo.

(a) Índice de Similitud de Raup-Crick

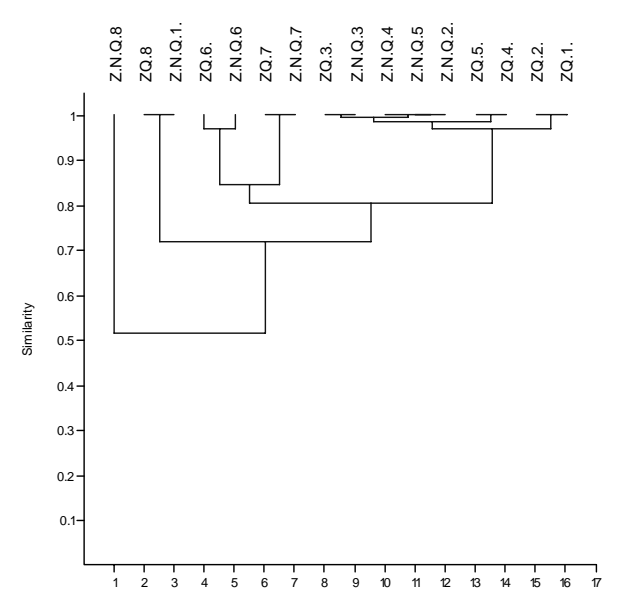

(b) Índice de Similitud de Morisita

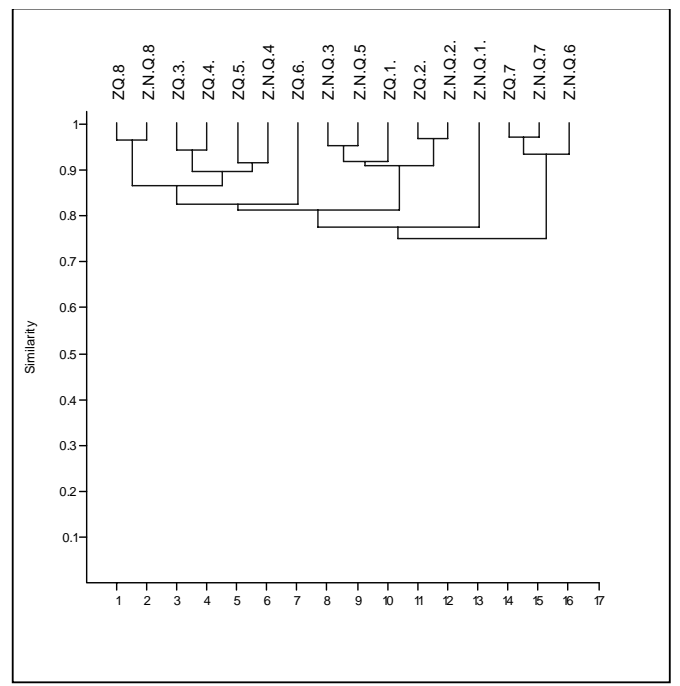


Figura 7. Macroartrópodos del grupo Funcional Parasitoides. Pachacayo. Agosto 2001 a Junio 2002.
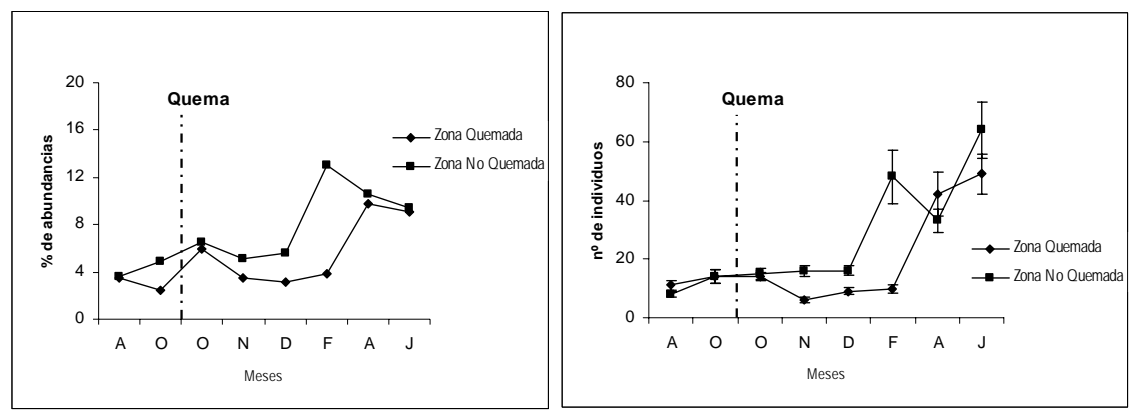

Figura 8. Porcentajes de Abundancia de los Microartrópodos del grupo Funcional Depredadores. Pachacayo. Agosto 2001 a Junio 2002.

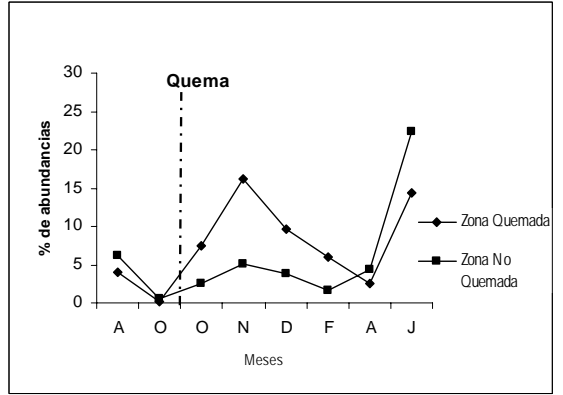

Figura 9. Evolución temporal de la Similitud de la Artropodofauna entre ambas zonas. Pachacayo. Agosto 2001 a Junio 2002.

(a)Índice de Similitud de Morisita.

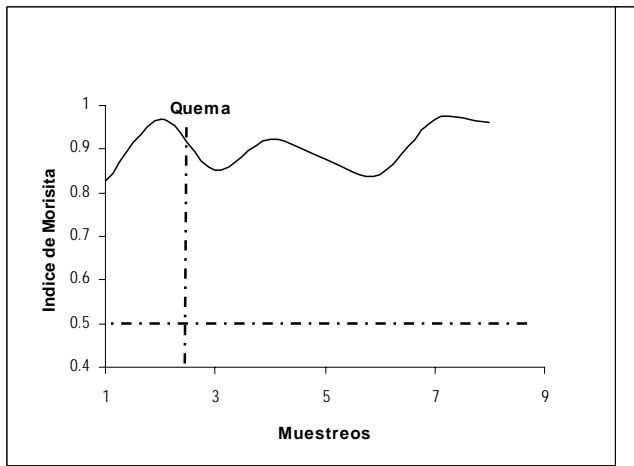

(b)Índice de similitud de Raup Crick.

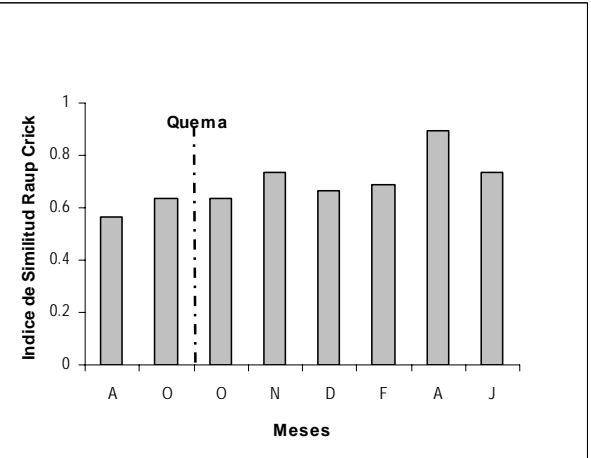

Figura 10. Evolución temporal de la Similitud de los Macroatrópodos y Microartrópodos entre ambas zonas. Pachacayo. Agosto 2001 a Junio 2002

(a) Macroartrópodos

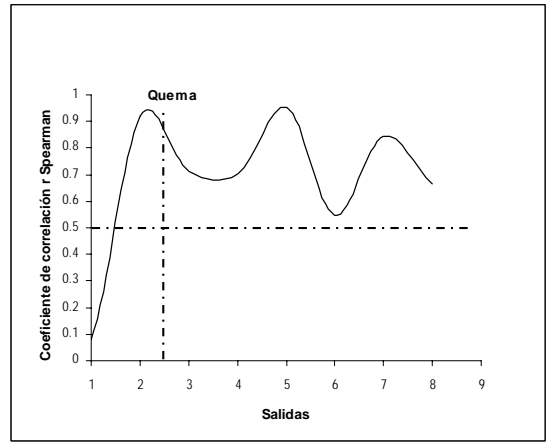

(b) Microartrópodos

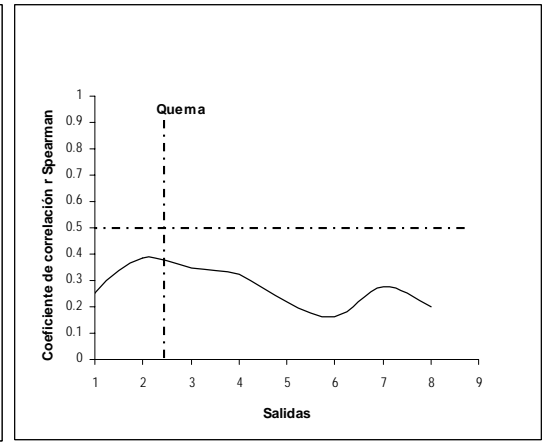


Figura 11. Evolución temporal de la Similitud de los Grupos Funcionales con y sin Entomonryidae-01 entre ambas zonas. Pachacayo. Agosto 2001 a Junio 2002

(a) Con Entomobryidae-01

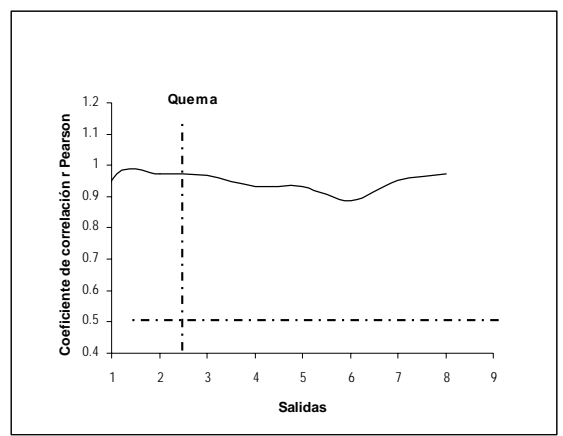

(b) Sin Entomobryidae-01

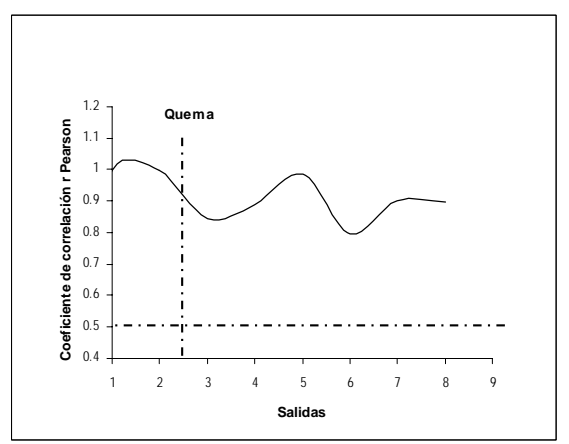

Anexo no 1 Abundancias de los Ordenes Taxonómicos de artrópodos terrestres colectados en Pachacayo (Jauja, Junín) durante Agosto de 2001 a Junio de 2002. Los números entre paréntesis son el número de morfoespecies contabilizadas en cada Orden.

\begin{tabular}{|c|c|c|c|}
\hline Clase & Orden & Zona Quemada (Total) & Zona No Quemada (Total) \\
\hline \multirow{5}{*}{ Arachnida } & Araneae & $177(11)$ & $165(15)$ \\
& Solifuga & $1(1)$ & $5(1)$ \\
& Gamasida & $248(2)$ & $289(1)$ \\
& Ixodida & $23(1)$ & $5(1)$ \\
& Actinedida & $105(6)$ & $63(4)$ \\
& Oribatida & $592(5)$ & $629(5)$ \\
\hline Collembola & Arthropleona & $4546(3)$ & $3636(3)$ \\
& Symphypleona & $896(1)$ & $1348(1)$ \\
\hline \multirow{5}{*}{ Insecta } & Orthoptera & $4(2)$ & $13(3)$ \\
& Dermaptera & 0 & $1(1)$ \\
& Psocoptera & 0 & $1(1)$ \\
& Thysanoptera & $2(1)$ & $3(2)$ \\
& Hemiptera (*) & $1083(28)$ & $1214(30)$ \\
& Coleoptera & $662(29)$ & $612(27)$ \\
& Diptera & $447(35)$ & $518(38)$ \\
& Hymenoptera & $162(39)$ & $221(39)$ \\
\hline
\end{tabular}

(*) Incluye subórdenes Homoptera y Heteroptera.

\footnotetext{
${ }^{1}$ Laboratorio de Ecología de Procesos. Departamento Académico de Biología. Universidad Nacional Agraria La Molina. Av. La Molina S/N. Apartado 12-056. Lima 12 - Perú. Dirección electrónica: licas78@yahoo.com

${ }^{2}$ Laboratorio de Ecología de Insectos. Departamento Académico de Biología. Universidad Nacional Agraria La Molina. Av. La Molina S/N. Apartado 12-056 Lima 12 - Perú. Dirección electrónica: acg@lamolina.edu.pe
} 